\title{
Interacción tripanosoma-vector-vertebrado y su relación con la sistemática y la epidemiología de la tripanosomiasis americana
}

\author{
Gustavo Adolfo Vallejo ${ }^{1}$,Felipe Guhl ${ }^{2}$, Julio César Carranza ${ }^{1}$, Omar Triana ${ }^{3}$, Gerardo Pérez ${ }^{4}$, \\ Paola Andrea Ortiz ${ }^{1}$, Dairo Humberto Marín ${ }^{1}$, Lina Marcela Villa ${ }^{1}$, Jazmín Suárez ${ }^{1}$, \\ Isaura Pilar Sánchez ${ }^{1}$, Ximena Pulido 1, Ingrid Bibiana Rodríguez ${ }^{1}$, Leyder Elena Lozano ${ }^{1}$, \\ Daniel Alfonso Urrea ${ }^{1}$, Fredy Arvey Rivera ${ }^{1}$, César Cuba-Cuba ${ }^{5}$, Jairo Alfonso Clavijo ${ }^{6}$ \\ 1 Laboratorio de Investigaciones en Parasitología Tropical, Facultad de Ciencias, Universidad del Tolima, \\ lbagué, Colombia. \\ 2 Centro de Investigaciones en Microbiología y Parasitología Tropical (CIMPAT), Universidad de los \\ Andes, Bogotá D.C., Colombia. \\ 3 Laboratorio de Chagas, Instituto de Biología, Facultad de Ciencias Exactas y Naturales, Universidad de \\ Antioquia, Medellín, Colombia. \\ ${ }^{4}$ Grupo de Investigación en Química de Proteínas, Departamento de Química, Universidad Nacional de \\ Colombia, Bogotá D.C., Colombia. \\ 5 Unidade de Parasitologia Médica e Biología de Vetores, Facultade de Medicina, Universidade de Brasilia, \\ Brasilia, DF, Brasil. \\ 6 Departamento de Matemáticas y Estadística, Facultad de Ciencias, Universidad del Tolima, Ibagué, \\ Colombia.
}

Introducción. Trypanosoma rangeli es la segunda especie de tripanosoma que infecta al hombre en América Latina. Se ha observado variabilidad en las características biológicas, bioquímicas y moleculares en diferentes aislamientos de este parásito.

Objetivo. Estudiar las características morfológicas y moleculares de cepas de T. rangeliaisladas de diferentes especies de Rhodnius e inoculadas en diferentes especies de vertebrados.

Materiales y métodos. Se utilizaron 19 cepas de $T$. rangeliaisladas de $R$. prolixus, $R$. pallescens y $R$. colombiensis en Colombia, $R$. ecuadoriensis en Perú y $R$. pallescens en Panamá. Se evaluó el polimorfismo de los tripomastigotes sanguíneos en ratones ICR y se estudió el pleomorfismo de la cepa P53 de T. rangeli KP1(-) inoculada en ratón, marsupial y canino. Se efectuó análisis de ADN polimorfo amplificado aleatorio en 12 cepas aisladas de cuatro especies de Rhodnius.

Resultados. Se observaron tres grupos discretos en la longitud total de los tripomastigotes sanguíneos y la cepa P53 presentó diferencias significativas en el tamaño de los tripomastigotes sanguíneos en ratón, marsupial y canino. El análisis de ADN polimorfo amplificado aleatorio mostró segregación de las cepas en dos ramas correspondientes a las cepas de $T$. rangeli $\mathrm{KP} 1(+)$ y $T$. rangeli KP1(-). De otra parte todos las cepas de $T$. rangeli KP1(-) se agruparon de acuerdo con las especies de Rhodnius de las cuales fueron aisladas.

Conclusión. Este es el primer estudio que revela una estrecha asociación entre cepas de $T$. rangeli y las especies de Rhodnius, confirmando que cada especie de Rhodnius transmite al hospedero vertebrado poblaciones del parásito con claras diferencias fenotípicas y genotípicas, lo cual soporta la evolución clonal de estas poblaciones.

Palabras clave: Trypanosoma, Rhodnius, tripanosomiasis/epidemiología, técnica del ADN polimorfo amplificado aleatorio (RAPD), enfermedad de Chagas.

Trypanosoma rangeli parasite-vector-vertebrate interactions and their relationship to the systematics and epidemiology of American trypanosomiasis

Introduction. Trypanosoma rangeli is a species of trypanosome second to $T$. cruzi, that is infective to humans in Latin America. Variability in the biological, biochemical and molecular characteristics between different isolates isolates of this parasite have been recorded.

Objective. Morphological and molecular characteristics were recorded from strains of $T$. rangeli 
that were isolated from different species of Rhodnius and maintained in different vertebrate species.

Materials and methods. Nineteen strains of T. rangeli were isolated from $R$. prolixus, $R$. pallescens and $R$. colombiensis in Colombia, $R$. ecuadoriensis in Peru and $R$. pallescens in Panama. Polymorphism of blood trypomastigotes in ICR mice was evaluated and pleomorphism of P53 strain of $T$. rangeli KP1(-) inoculated in mouse, marsupial and canine was studied. RAPD analysis (randomly amplified polymorphic DNA analysis) of 12 strains isolated from four species of Rhodnius was performed.

Result. Based on the total length of blood trypomastigotes, three discrete groups were observed. The P53 strain showed significant differences in the size of blood trypomastigotes in mouse, marsupial and canine. RAPD analysis showed that the strains segregated into two branches corresponding to strains of $T$. rangeli $\mathrm{KP} 1(+)$ and $T$. rangeli $\mathrm{KP} 1(-)$. All strains of $T$. rangeli $\mathrm{KP} 1$ (-) clustered according to the species of Rhodnius from which they were isolated .

Conclusion. These data reveal, for the first time, a close association amongst $T$. rangeli strains and Rhodnius species, confirming that each species of Rhodnius transmits to vertebrate hosts a parasite population with clear phenotypic and genotypic differences. This is further evidence that supports the concept of clonal evolution of these parasites.

Key words: Trypanosoma, Rhodnius, trypanosomiasis/epidemiology, random amplified polymorphic DNA technique, Chagas disease.

Trypanosoma rangeli es un interesante parásito no patógeno para el hombre, que exhibe particulares características biológicas, entre las cuales se destacan la patogenicidad del parásito para el vector (1-3), la susceptibilidad de las especies de Rhodnius a cepas de $T$. rangeli del mismo origen geográfico $(4,5)$, la reacción serológica cruzada de $T$. rangelicon $T$. cruzi $(6,7)$ y la resistencia de los flagelados a la lisis mediada por el complemento (8), entre otras. Por ello, durante las últimas décadas, $T$. rangeli ha sido objeto de estudio por parte de varios grupos de investigación que han encontrado en este parásito un importante modelo para conocer los procesos coevolutivos entre parásito-vector y para estudiar la dinámica de la transmisión de subpoblaciones de $T$. rangeli al hospedero vertebrado (9). De otra parte, existe interés en desarrollar métodos para diferenciar $T$. cruzi de $T$. rangeli, debido a que en la mayoría de los países de América Latina estas dos especies se encuentran infectando los mismos vertebrados y vectores, generando dificultades en el diagnóstico morfológico de las

\footnotetext{
Correspondencia:

Gustavo Adolfo Vallejo, Laboratorio de Investigaciones en Parasitología Tropical, Facultad de Ciencias, Universidad del Tolima, Ibagué, Tolima, Colombia. A.A. No. 546. Telfax +(57) 82669176.

gvallejo@ut.edu.co
}

Recibido: 23/06/06; aceptado: 14/07/06 dos especies (10) o en el diagnóstico serológico de la infección en los vertebrados $(6,7,11)$.

La variabilidad de las poblaciones de $T$. rangeli se ha demostrado en las últimas décadas mediante estudios de minisatélites de ADN, los cuales mostraron diferencias genéticas entre las cepas de Santa Catarina y las aisladas en Honduras, Colombia y Venezuela (12). El análisis de polimorfismos del ADN mitocondrial mostró que el minicírculo de kDNA denominado KP1 se encontró asociado con las cepas de $T$. rangeli aisladas en Colombia, Honduras y Venezuela, pero no en las cepas aisladas en Santa Catarina al sureste de Brasil (13). Una divergencia genética similar entre estos dos grupos se demostró usando electroforesis de isoenzimas y AP-PCR (14). Una conclusión similar se obtuvo con el análisis del cariotipo molecular de varias cepas de $T$. rangeli, revelando la existencia de polimorfismo cromosómico (15).

Utilizando amplificación del kDNA, en Colombia y en otros países de América Latina se han detectado dos grupos de $T$. rangeli molecularmente diferentes. Un grupo de cepas aisladas de Rhodnius prolixus presentan tres clases de minicírculos de kDNA denominados KP1, KP2 y KP3 ( $T$. rangeli $\mathrm{KP} 1+$ ), mientras que las cepas aisladas de $R$. colombiensis presentan solamente dos clases denominadas KP2 y KP3 (T. rangeli KP1-) $(9,16-18)$. 
Recientemente se caracterizaron 18 cepas de $T$. rangeli aisladas de glándulas salivares de $R$. colombiensis, $R$. pallescens y $R$. prolixus (18) utilizando dos marcadores independientes: kDNA (17) y el espaciador intergénico del gen mini-exón (19). Los autores observaron que el producto de 380 pb de amplificación del mini-exón siempre se presentó asociado con los productos obtenidos de los minicírculos KP2 Y KP3 de kDNA y el producto de $340 \mathrm{pb}$ del mini-exón siempre se presentó asociado con los productos de los minicírculos KP1, KP2 Y KP3. Existen dos hipótesis alternativas sobre el origen de los dos grupos de $T$. rangeli; por un lado podría tratarse de grupos con evolución clonal, en la cual la reproducción sexual sería rara o inexistente, o se trataría de un proceso de especiación críptica.

Se encontró asociación coevolutiva entre las cepas de $T$. rangeli KP1(-), las cuales circulan en la cordillera de los Andes en los vectores del grupo "pallescens", representados por $R$. pallescens, $R$. colombiensis y $R$. ecuadoriensis, y las cepas de $T$. rangeli KP1(+), las cuales circulan al oriente de la cordillera de los Andes en los vectores del grupo "prolixus", representados por $R$. prolixus y R. neglectus (9).

Nuestro interés fue conocer el grado de divergencia genética existente entre los grupos de $T$. rangeli KP1(+) y $T$. rangeli KP1(-). Por esta razón, en el presente trabajo se analizaron las características morfológicas de los tripomastigotes sanguíneos y las características genéticas mediante análisis de RAPD en cepas de $T$. rangeli aisladas de $R$. pallescens, $R$. colombiensis, $R$. ecudoriensis y $R$. prolixus para verificar si los polimorfismos observados en las poblaciones de este parásito están asociados con los vectores que las transmiten, de manera que estos actuarían como filtros biológicos seleccionando las diferentes poblaciones del parásito.

\section{Materiales y métodos}

\section{Aislamiento y cultivo de los parásitos}

Se utilizaron 19 cepas de $T$. rangeli. Las cepas Perú 1 , Perú $1 \mathrm{~A}$ y Perú $2 \mathrm{~A}$ se aislaron de las glándulas salivares naturalmente infectadas de $R$. ecuadoriensis domiciliado en el departamento de la Libertad en el norte del Perú; las cepas P53, Trs, Rcol, 2715 y 044 se aislaron de $R$. colombiensis silvestre en el departamento del Tolima, región central de Colombia; las cepas Gal 47, Gal 57, SO28, SO48 se aislaron de $R$. pallescens silvestre en el departamento de Sucre, norte de Colombia; la cepa 0401 se aisló de $R$. pallescens silvestre en el departamento del Tolima, Colombia; la cepa GMLLC se aisló de $R$. pallescens en Panamá; las cepas 444, 1545, 3123 se aislaron de $R$. prolixus domiciliado en el departamento de Boyacá, Colombia, y las cepas P19 y Choachí se aislaron de $R$. prolixus domiciliado en los departamentos de Norte de Santander y Cundinamarca, Colombia. Los tripomastigotes metacíclicos de las glándulas salivares de cada vector se inocularon intraperitonealmente en ratones ICR. Se efectuaron hemocultivos a partir de los ratones positivos en el quinto día posinoculación y las cepas aisladas se mantuvieron a $28^{\circ} \mathrm{C}$ mediante pasajes semanales en medio NNN con LIT suplementado con $10 \%$ de suero fetal bovino inactivado. Todas las cepas aisladas de $R$. ecuadoriensis, $R$. colombiensis y $R$. pallescens se caracterizaron como $T$. rangeli KP1(-), y las cepas aisladas de $R$. prolixus como $T$. rangeli $\mathrm{KP} 1(+)$ mediante la amplificación de kDNA y del espaciador intergénico del gen min-iexón $(17,19)$.

\section{Estudio morfológico de los tripomastigotes sanguíneos en ratones ICR}

Grupos de tres ratones ICR machos de \pm 20 gramos se inocularon con $5 \times 10^{6}$ epimastigotes de las cepas de cultivo de $T$. rangeli $\mathrm{KP} 1(-)$. Otros grupos de tres ratones ICR machos se inocularon con 400.000 tripomastigotes metacíclicos de cada cepa de $T$. rangeli $\mathrm{KP} 1(+)$. Los ratones se examinaron a partir del tercer día de inoculación, se observó la parasitemia y se dibujaron en cámara lúcida 30 formas sanguíneas circulantes de cada una de las cepas en el quinto día posinoculación. En cada una de las formas dibujadas se midió la longitud total, la longitud del flagelo, las distancias del núcleo al extremo posterior y al extremo anterior, los índices nucleares y los índices del cinetoplasto $(20,21)$. Se realizaron estimaciones de las medias de la longitud total 
de los tripomastigotes sanguíneos de las cepas de $T$. rangeli en el quinto día posinfección con los respectivos intervalos de $95 \%$ de confianza y se efectuó análisis multivariado de varianza (MANOVA) utilizando el programa STATISTICA V4.5.

\section{Estudio morfológico de Trypanosoma rangeli en diferentes hospederos vertebrados}

Para evaluar pleomorfismos de las formas sanguíneas se seleccionó la cepa P53 de $T$. rangeliKP1(-) para infectar dos individuos de Mus musculus, Didelphis marsupialis y Canis familiaris, a los que se les inocularon formas de cultivo que contenían $2 \times 10^{6}$ parásitos por gramo de peso corporal. Los tripomastigotes sanguíneos se aislaron de la sangre de cada uno de los mamíferos, concentrándolos por centrifugación en tubo capilar, extendiéndolos y coloreándolos con Giemsa. Se dibujaron 30 tripomastigotes sanguíneos circulantes en ratón, canino y didélfido durante el tercer día posinoculación. A cada una de las formas dibujadas se les efectuó la toma de las medidas morfométricas y se realizaron estimaciones de las medias de la longitud total de los tripomastigotes sanguíneos de la cepa P53 de $T$. rangelien el tercer día posinfección en cada uno de los mamíferos utilizados con los respectivos intervalos de 95\% de confianza; se efectuó análisis multivariado de varianza (MANOVA) utilizando el programa STATISTICA V4.5.

\section{Purificación de ADN y obtención de RAPD}

El ADN genómico se extrajo de los cultivos de parásitos mediante el método de fenol-cloroformo seguido de precipitación con etanol y acetato de sodio. La concentración del ADN se determinó por electroforesis mediante comparación con patrones de concentración conocidos. Para la obtención del ADN polimorfo amplificado aleatorio (RAPD) se ensayaron 12 iniciadores a partir de los cuales se seleccionaron OPBG-02 (5'GGAAAGCCCA-3') y OPBG-013 (5'-GGTTGGGC CA-3') (Operon Technologies, Inc.), los que permitieron obtener los patrones más discriminantes para analizar todas las cepas. Para las amplificaciones se utilizó un volumen final de reacción de $20 \mu \mathrm{l}$ que contenía $2 \mathrm{ml}$ de buffer de Taq Polimerasa 10X (INVITROGEN), una mezcla de dNTPs $200 \mathrm{mM}$ dNTPs, $\mathrm{MgCl}_{2} 3,5 \mathrm{mM}, \mathrm{KCL}$ $25 \mathrm{mM}$ y $25 \mathrm{mM}$ del iniciador , 1 unidad de Taq DNA polimerasa (INVITROGEN) y $10 \mathrm{ng}$ de ADN. La reacción en cadena de la polimerasa (PCR) se corrió en una termociclador M.J. Research PTC150-16 y se sometió a 35 ciclos de amplificación. Los perfiles de temperatura para denaturación del ADN, hibridación y extensión del iniciador fueron $95^{\circ} \mathrm{C}$ por un minuto (con un tiempo inicial de 5 $\min$ ), $30^{\circ} \mathrm{C}$ por un minuto y $72^{\circ} \mathrm{C}$ por un minuto, respectivamente, y una extensión final de 5 minutos. Los productos amplificados fueron separados en geles de poliacrilamida al $6 \%$ y coloreados con nitrato de plata (22). Los perfiles de RAPD se usaron para la construcción de una matriz binaria de acuerdo con la presencia (1) 0 ausencia (0) de las bandas. El análisis se efectuó con el programa RAPDPLOT versión 3.0 y se calculó la matriz de distancias a partir del índice de bandas compartidas (1-MATCH). El índice de bandas compartidas (M) se calculó usando la formula: $\mathrm{M}=\mathrm{NAB} / \mathrm{NT}$, donde NAB es el número total de apareamientos entre los individuos $A$ y $B$ y NT es el número total de loci registrados (23). Con base en los resultados de los perfiles de RAPD se construyó un dendrograma por el método UPGMA (Unweighted Pair-Group Method with Arithmetic Means) utilizando un bootstrap con 100 pseudoréplicas. Las distancias entre los grupos de parásitos se obtuvieron con el programa RAPDIST, con el cual se compararon las distancias genéticas de Nei (24), y se comprobó la consistencia de los datos de RAPD que soportan las ramas del dendrograma usando análisis de bootstrap con 100 pseudoréplicas.

\section{Resultados}

\section{Morfología de los tripomastigotes sanguíneos en ratones ICR}

Los resultados del estudio morfológico de 10 cepas de los tripomastigotes sanguíneos de $T$. rangeli en la sangre de ratones infectados experimentalmente mostró un elevado grado de polimorfismo de los parásitos en el quinto día de infección. Se observaron promedios en las longitudes totales de los tripomastigotes desde 32 hasta $39 \mu \mathrm{m}$ mostrando diferencias significativas entre las diferentes cepas analizadas (figura 1). 


\section{Morfología de Trypanosoma rangeli en diferentes hospederos vertebrados}

Se observó que la cepa P53 de $T$. rangeli presentó diferencias estadísticamente significativas entre las tres especies de mamíferos. Los tripomastigotes sanguíneos fueron más cortos en ratón que en sangre de Didelphis y aun más cortos que los observados en sangre de perro (figura 2).

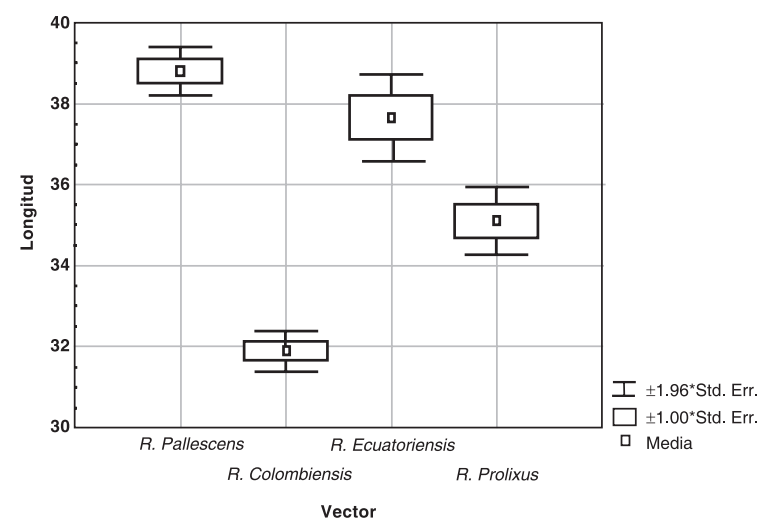

Figura 1. Comparación de las medias de la longitud total de 30 tripomastigotes sanguíneos de cada una de cuatro cepas estudiadas en ratones ICR en el quinto día posinoculación. Se compararon las cepas Gal 57, aislada de R. pallescens, P53, aislada de $R$. colombiensis, Perú 1, aislada de $R$. ecuadoriensis, y $\mathrm{P} 19$, aislada de $R$. prolixus.

\section{Obtención y análisis de RAPD}

La figura 3 muestra uno de los geles de poliacrilamida al $6 \%$ con los productos amplificados y coloreados con nitrato de plata. Con base en los resultados de los perfiles de RAPD se construyó un dendrograma por el método UPGMA (Unweighted Pair-Group Method with Arithmetic Means) utilizando un bootstrap con 100 pseudoréplicas (figura 4). De acuerdo con el

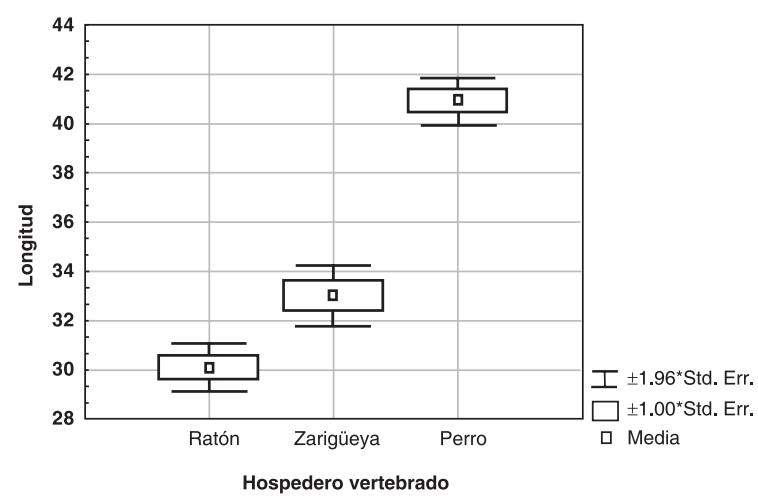

Figura 2. Comparación de las medias de la longitud total de 30 tripomastigotes snaguíneos de la cepa de T. rangeli P53 detectada en el tercer día posinoculación en ratón, zarigüeya y perro.

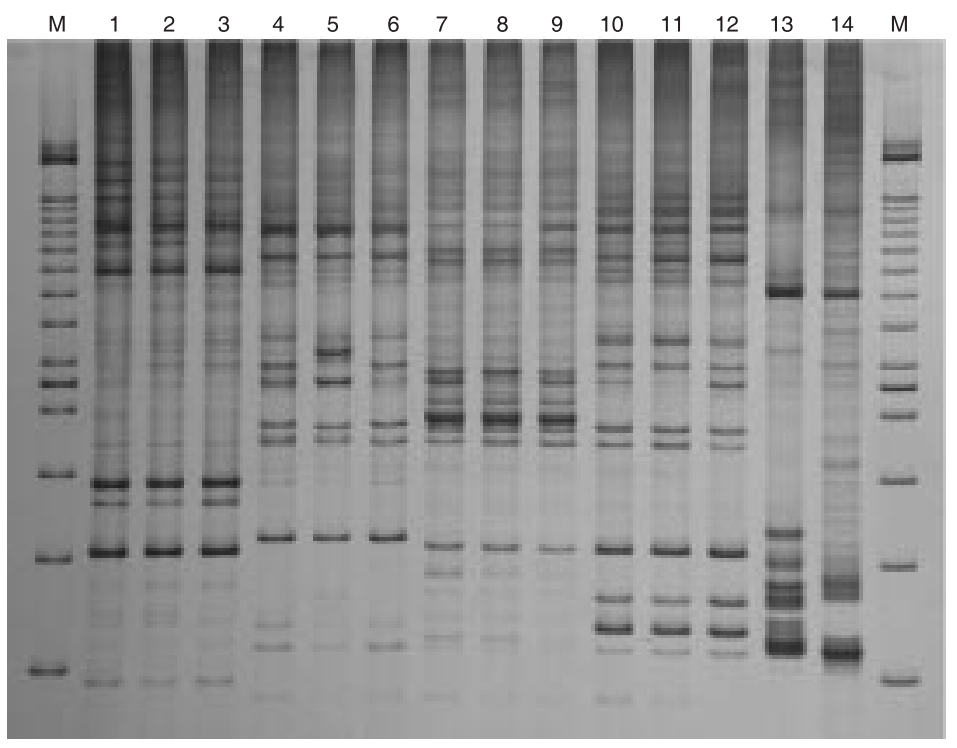

Figura 3. Perfiles de RAPD obtenidos a partir de 12 cepas de $T$. rangelicon el primer OPBG13. M corresponde al marcador de peso molecular. Las canaletas 1,2 y 3 corresponden a las cepas de $T$. rangeli $444,1545,3123$, respectivamente, aisladas de $R$. prolixus. Las canaletas 4,5 y 6 , corresponden a las cepas de $T$. rangeli Gal47, SO28 y SO48, respectivamente, aisladas de $R$. pallescens. Las canaletas 7, 8 y 9 corresponden a las cepas de $T$. rangeli Perú1, Perú1A y Perú2A, respectivamente, aisladas de $R$. ecuadoriensis. Las canaletas 10, 11 y 12 corresponden a las cepas de $T$. rangeli P53, TrS y Rcol, respectivamente, aisladas de $R$. colombiensis. La canaleta 13 corresponde a una cepa de $T$. cruzi I y la canaleta 14 corresponde a una cepa de $T$. cruzi II. 
dendrograma, las doce cepas de $T$. rangeli se agruparon en dos ramas. Las cepas 3123,444 , y 1545 aisladas de $R$. prolixus, caracterizadas como $T$. rangeli $\mathrm{KP} 1(+)$, formaron una rama y las otras nueve cepas aisladas de las glándulas salivares del grupo "pallescens", conformado por $R$. pallescens (SO28, Gal 47, SO48), $R$. colombiensis (Rcol, P53, Trs) y $R$. ecuadoriensis (Perú 2A, Perú 1A, Perú 1), caracterizadas como T. rangeliKP1(-), formaron una segunda rama con tres subramas que correspondieron exactamente a cada una a las cepas aisladas de $R$. pallescens, $R$. colombiensis y $R$. ecuadoriensis. La figura 5 muestra las distancias de Nei entre los grupos de parásitos obtenidas con el programa RAPDIST.

\section{Discusión}

La interacción entre las subpoblaciones de tripanosomas con diferentes especies de triatominos, así como los mecanismos que regulan la transmisión, son importantes elementos para la documentación de la epidemiología de la tripanosomiasis americana. A partir de este enfoque será posible establecer cuáles subpoblaciones de tripanosomas son transmitidas por los vectores en una determinada área geográfica. De otra parte será igualmente posible conocer si existen diferencias en la susceptibilidad a la infección de los hospederos vertebrados. Adicionalmente, los estudios de la interacción tripanosoma-triatomino permitirán identificar procesos coevolutivos para fortalecer las hipótesis

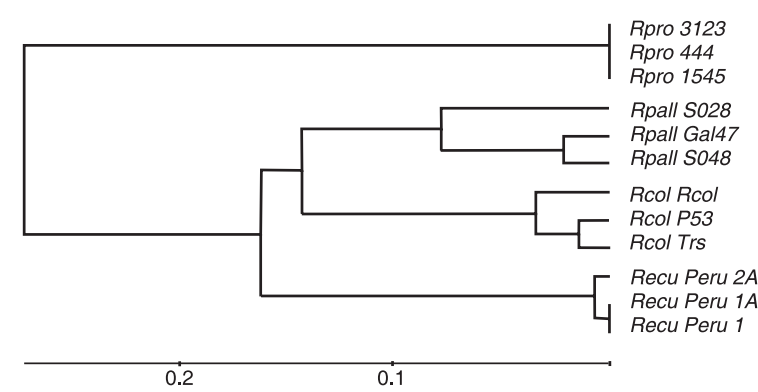

Figura 4. Fenograma generado por UPGMA basado en los perfiles de RAPD de 12 cepas de Trypanosoma rangeli aisladas de Rhodnius prolixus (Rpro), R. pallescens (Rpall), $R$. colombiensis (Rcol) y R. ecuadoriensis (Recu). Los números de la escala horizontal fueron derivados de la distancia calculada con el programa RAPDPLOT.

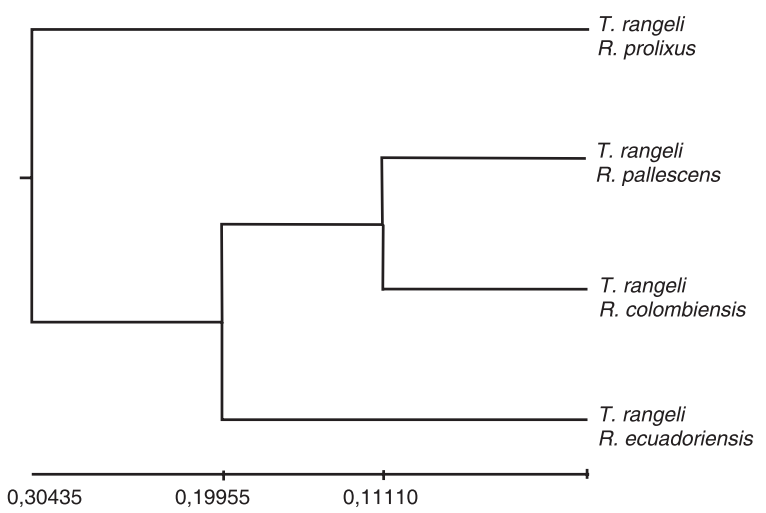

Figura 5. Dendrograma generado por RAPDDIST que muestra las distancias genéticas de Nei entre las cepas de $T$. rangeli aisladas de $R$. prolixus, $R$. pallescens, $R$. colombiensis y $R$. ecuadoriensis usando el algoritmo UPGMA y bootstrap de 100 seudoréplicas.

acerca de la filogenia y dispersión de los parásitos y de los vectores, contribuyendo así a aclarar la posición taxonómica de las especies semejantes a $T$. cruzi o a $T$. rangeli en América.

Se ha demostrado que las cepas de $T$. rangeli aisladas de las especies de Rhodnius del grupo «pallescens» (R. pallescens, $R$. colombiensis y $R$. ecuadoriensis) denominadas (KP1-) son genéticamente divergentes de las cepas aisladas del grupo «prolixus» (prolixus, neglectus) denominadas (KP1+) $(9,17,18)$, lo que indica una posible asociación evolutiva entre las subpoblaciones de $T$. rangeli $y$ los grupos de especies de Rhodnius.

La relación entre la diversidad genética del parásito y su transmisibilidad vectorial no está completamente entendida, pues se ha mostrado que las poblaciones de $T$. cruzi y $T$. rangeli difieren en su habilidad para sobrevivir, multiplicarse y diferenciarse en el insecto vector. También se ha mostrado que en una misma área geográfica existen diferencias significativas entre la proporción de genotipos de $T$. cruzi y $T$. rangeli detectados en los vectores y en los mamíferos. Estas diferencias podrían explicarse por la transmisión selectiva de algunos genotipos por parte de los vectores locales, que actuarían como filtros biológicos del parásito. Se ha demostrado que en Colombia $R$. colombiensis 
puede infectarse con $T$. rangeli KP1(-) y KP1(+), pero sólo transmite a KP1(-) a través de la picadura y que $R$. prolixus se infecta con $T$. rangeli KP1(-) y $\mathrm{KP} 1(+)$, pero solo transmite por picadura a la subpoblación $\mathrm{KP} 1(+)(17,18)$. Estos resultados demuestran que $R$. prolixus es susceptible a la infección de $T$. rangeli KP1(+), pero presenta una respuesta refractaria a la invasión de cepas de $T$. rangeli KP1(-) aisladas de $R$. pallescens, $R$. colombiensis y $R$. ecuadoriensis. El comportamiento diferencial en la transmisión de estas dos subpoblaciones se relaciona con la activación de la respuesta inmune del vector, en la que factores intrínsecos como la producción de hemocitos, nódulos y factores tripanolíticos limitan la infección parasitaria (25). Recientemente se detectó en la hemolinfa de $R$. prolixus un factor tripanolítico contra las cepas de $T$. rangeli KP1(-) que corresponde a una proteína termolábil, la cual sería un factor inmunológico para que $R$. prolixus actúe como filtro biológico de subpoblaciones de T. rangeli KP1(-) (datos no publicados).

En el presente trabajo se detectó un elevado polimorfismo de los tripomastigotes sanguíneos medidos en el quinto día posinoculación. El tamaño promedio de las cepas varió entre 32 y $39 \mu \mathrm{m}$, mostrando en general la existencia de grupos discretos (figura 1). Es interesante el hecho de que tres cepas aisladas de $R$. pallescens mostraron siempre tamaños promedios muy cercanos a los $40 \mu \mathrm{m}$ (datos no mostrados), mientras que las cepas asiladas de $R$. colombiensis, $R$. ecuadoriensis y $R$. prolixus mostraron tamaños variables entre 32 y $39 \mu \mathrm{m}$. Esta observación sugiere la existencia de por lo menos dos grupos morfológicos de $T$. rangeli. Por otro lado, estos polimorfismos podrían ser el resultado de la transmisión selectiva de subpoblaciones del parásito, de manera que en las glándulas salivares del vector se desarrollaría cada vez una subpoblación con características morfológicas diferentes.

Dentro de una misma especie de tripanosoma pueden encontrarse variaciones morfológicas significativas que pueden conducir a la separación de una misma especie en dos o más especies diferentes (21). Varios autores han señalado pleomorfismos o variaciones morfológicas de $T$. rangelicuando se infectan diferentes vertebrados con un mismo aislamiento. El pleomorfismo de los tripomastigotes sanguíneos detectado en el presente trabajo en ratón, Didelphis y perro al tercer día posinoculación revela que la longitud total de estas formas de $T$. rangeli sería dependiente del vertebrado en el cual se examina (figura 2). Estos resultados preliminares indican que probablemente el vertebrado también selecciona diferentes subpoblaciones del parásito, o que la morfología de $T$. rangeli sería dependiente de la respuesta inmune del hospedero vertebrado. El hallazgo de este carácter pleomorfico en $T$. rangeli podría contribuir a aclarar la posición taxonómica de los tripanosomas semejantes a $T$. rangeli, tales como $T$. (H.) mesnilbrimontí, $T$. (H.) preguici, T. (H.) myrmecophague, $T$. (H.) mycetae, T. (H.) diasi, $T$, (H.) cebus, T. (H.) saimiri, y $T$. $(H$.$) advieri, entre otros, pues como se sabe, estos$ parásitos fueron clasificados como especies diferentes con base en diferencias de tamaño al ser comparados con cepas de $T$. rangeli aisladas de $R$. prolixus.

Los resultados de la caracterización molecular preliminar mediante RAPD de las cepas de $T$. rangeli (figura 4) muestran una clara divergencia entre el grupo de cepas KP1(+) y KP1(-), indicando la existencia de cuatro grupos de $T$. rangeli genéticamente divergentes y asociados con $R$. prolixus, $R$. pallescens, $R$. colombiensis y $R$. ecuadoriensis. Estos grupos difieren de los cuatro grupos de $T$. rangeli previamente detectados por Maia da Silva et al. (26), quienes utilizando análisis de RAPD encontraron un grupo formado por cepas de T. rangeli de Colombia, Venezuela, Honduras, la Isla de Marajó, Pará y Rondonia en Brasil, un segundo grupo formado por las cepas aisladas en Acre y Pará (Brasil), un tercer grupo formado por cepas aisladas en Panamá y el Salvador y un cuarto grupo formado por la cepa SC58 aislada en Santa Catarina (Brasil). Los resultados del presente trabajo, en conjunto con los de otros autores (26), indican que $T$. rangeli está formado por varios grupos genéticamente divergentes, probablemente asociados a diferentes especies de Rhodnius. Posteriores estudios filogenéticos en el grupo de cepas KP1(-), utilizando otros marcadores moleculares, podrían indicar si estas 
cepas evolucionaron de norte a sur en el mismo sentido que se propone la evolución del cline conformado por las especies de vectores del grupo "pallescens". Por otro lado, los estudios filogenéticos en el grupo de $T$. rangeli $\mathrm{KP} 1(+)$ podrían arrojar información sobre la evolución de los parásitos y sus vectores.

Las distancias genéticas de Nei observadas por medio del análisis de isoenzimas en especies diferentes de un mismo género varían entre 0,162 y 0,3 en la mayoría de las especies (27). Las distancias obtenidas entre las subpoblaciones de T.rangelien el presente trabajo (figura 5) indicaron que los grupos más próximos constituídos por las cepas aisladas de $R$. pallescens y $R$. colombiensis presentaron una distancia genética de Nei de 0,11110; sin embargo, las cepas aisladas de $R$. ecuadoriensis presentaron una distancia genética de 0,19955 con relación al grupo R.pallescens- $R$. colombiensis; por otro lado, el cálculo de la distancia genética de Nei entre poblaciones de $T$. rangeli con el empleo de RAPDIST mostró que las distancias entre $T$. rangeli $\mathrm{KP} 1(-)$ y $\mathrm{T}$. rangeli KP1(+) fueron de 0,30435, lo cual indica una gran divergencia genética entre estos dos grupos, semejante a la observada en especies diferentes de otros organismos (27).

Los resultados de la presente investigación señalan que la interacción $T$. rangeli-vector es un importante modelo, en el cual cada vector del género Rhodnius transmite subpoblaciones del parásito fenotípica y genotípicamente divergentes y que, por lo tanto, los vectores actuarían como filtros biológicos de dichas subpoblaciones. Es posible que ocurran fenómenos semejantes en la transmisión del agente causal de la enfermedad de Chagas, pues recientemente se han descrito seis subpoblaciones de T. cruzi (T. cruzil, T. cruzi Ila, Ilb, Ilc, Ild y Ile) $(28,29)$, que interactúan con más de 100 especies de triatominos en diferentes regiones de América Latina.

\section{Conflicto de intereses}

Los autores del presente artículo declaramos que no teníamos conflictos de intereses de orden académico, institucional $u$ operacional en el momento de realización de la investigación.

\section{Financiación}

El presente trabajo recibió financiación de las siguientes instituciones: Instituto Colombiano "Francisco José de Caldas" (Colciencias), proyecto 1105-05-16919; Chagas Disease Intervention Activities-European Community (CDIAEC), contrato No. ICA4-CT-2003-10049; SSA-EC: Trypanosomiasis Update y Fondo de Investigaciones de la Universidad del Tolima.

\section{Referencias}

1. Marinkelle CJ. Pathogenicity of Trypanosoma rangeli for Rhodnius prolixus Stal in nature. J Med Entomol 1968;5:497-9.

2. Watkins R. Trypanosoma rangeli: effect on excretion in Rhodnius prolixus. J Invertebr Pathol 1971;17:67-71.

3. Watkins R. Histology of Rhodnius prolixus infected with Trypanosoma rangeli. J Invertebr Pathol 1971;17:59-66.

4. D'Alessandro-Bacigalupo A, Gore-Saravia N. Trypanosoma rangeli. En: Kreier JP, editor. Parasitic protozoa. London: Academic Press; 1992.p.1-54.

5. D'Alessandro-Bacigalupo A, Gore-Saravia N. Trypanosoma rangeli. En: Gilles HM, editor. Protozoal diseases. Oxford: Oxford University Press; 1999. p.398-412.

6. Guhl F, Hudson L, Marinkelle CJ, Morgan S, Jaramillo C. Antibody response to experimental Trypanosoma rangeli infection and its implications for immunodiagnosis of South American trypanosomiasis. Acta Trop 1985;42:311-8.

7. Guhl F, Hudson L, Marinkelle CJ, Jaramillo CA, Bridge D. Clinical Trypanosoma rangeli infection as a complication of Chagas' disease. Parasitology 1987;94:475-84

8. Guhl F, Vallejo GA. Trypanosoma (Herpetosoma) rangeli Tejera, 1920: an updated review. Mem Inst Oswaldo Cruz 2003;98:435-42.

9. Urrea DA, Carranza JC, Cuba CA, GurgelGonçalves R, Guhl F, Schofield CJ, et al. Molecular characterisation of Trypanosoma rangeli strains isolated from Rhodnius ecuadoriensis in Peru, $R$. colombiensis in Colombia and $R$. pallescens in Panama, supports a co-evolutionary association between parasites and vectors. Infect Genet Evol 2005;5:123-9.

10. Vallejo GA, Marinkelle CJ, Guhl F, De Sánchez N. Comportamiento de la infección y diferenciación morfológica entre Trypanosoma cruzi y $T$. rangeli en el intestino del vector Rhodnius prolixus. Rev Bras Biol 1988;48:577-87. 
11. Guhl F, Jaramillo C, Carranza JC, Vallejo GA. Molecular characterization and diagnosis of Trypanosoma cruzi and T. rangeli. Arch Med Res 2002;33:362-70.

12. Macedo AM, Vallejo GA, Chiari E, Pena SD. DNA fingerprinting reveals relationships between strains of Trypanosoma rangeli and Trypanosoma cruzi. En: Pena SD, Chakraborty R, Epplen JT, Jeffreys AJ, editors. DNA fingerprinting: state of the Science. Basel, Switzerland: Birkhauser Verlag: 1993. p.321-9.

13. Vallejo GA, Macedo AM, Chiari E, Pena SD. Kinetoplast DNA from Trypanosoma rangeli contains two distinct classes of minicircle with different size and molecular organization. Mol Biochem Parasitol 1994;67:245-53.

14. Steindel M, Dias-Neto E, Pinto CJ, Grisard E, Menezes C, Murta SM, et al. Randomly amplified polymorphic DNA (RAPD) and isoenzyme analysis of Trypanosoma rangeli strains. J Eukaryot Microbiol 1994;41:261-7.

15. Toaldo CB, Steindel M, Sousa MA, Tavares CC. Molecular karyotype and chromosomal localization of genes encoding 0-tubulina, cisteina proteinase, HSP 70 and actin in Trypanosoma rangeli. Mem Inst Oswaldo Cruz 2001;96:113-21.

16. Vallejo GA, Guhl F, Chiari E, Macedo AM. Specie specific detection of Trypanosoma cruzi and Trypansoma rangeli in vector and mammalian hosts by polymerase chain reaction amplification of kinetoplast minicircle DNA. Acta Trop 1999;72:203-12.

17. Vallejo GA, Guhl F, Carranza JC, Lozano LE, Sánchez JL, Jaramillo JC, et al. kDNA markers define two major Trypanosoma rangeli lineages in Latin America. Acta Trop 2002;81:77-82.

18. Vallejo GA, Guhl F, Carranza JC, Moreno J, Triana O, Grisard EC. Parity between kinetoplast DNA and miniexon gene sequences supports either clonal evolution or speciation in Trypanosoma rangeli strains isolated from Rhodnius colombiensis, $R$. pallescens and $R$. prolixus in Colombia. Infect Genet Evol 2003;3:39-45.

19. Grisard EC, Campbell DA, Romanha AJ. Mini-exon gene sequence polymorphism among Trypanosoma rangeli strains isolated from distinct geographical regions. Parasitology 1999;118:375-82.

20. Hoare CA. Herpetosoma from man and other mammals. En: The trypanosomes of mammals: a zoological monograph. Oxford: Blackwell Scientific Publications 1972.p.288-314

21. Ziccardi M, Lourenco-de-Oliveira R. Polymorphism in trypomastigotes of Trypanosoma (Megatrypanum) minasense in the blood of experimentally infected squirrel monkey and marmosets. Mem Inst Oswaldo Cruz 1999;94:649-53.

22. Sanguinetti CJ, Dias-Neto E, Simpson AJ. Rapid silver staining and recovery of PCR products separated on polyacrylamide gels. Biotechniques 1994;17:914-21.

23. Black WC. Statistical analysis of arbitrarily primed PCR patterns in molecular taxonomic studies. En: Clap CL, editor. Methods in molecular biology. Species diagnostics protocols: PCR and other nucleic acid methods. Vol. 50. Totowa, NJ: Humana Press; 1995. p.39-55.

24. Nei M. Estimation of average heterozygocity and genetic distance from a small number of individuals. Genetics 1978;89:583-90

25. Sánchez IP, Pulido XC, Carranza JC, Triana $\mathbf{O}$, Vallejo GA. Inmunidad natural de Rhodnius prolixus (Hemiptera: Reduviidae: Triatominae) frente a la infección con Trypanosoma (Herpetosoma) rangeli KP1(-) aislados de Rhodnius pallescens, $R$. colombiensis y $R$. ecuadoriensis. Revista de la Asociación Colombiana de Ciencias Biológicas 2005;17:108-18

26. Maia da Silva F, Rodrigues AC, Campaner M, Takata CS, Brigido MC, Junqueira AC, et al. Randomly amplified polymorphic DNA analysis of Trypanosoma rangeli and allied species from human, monkeys and other sylvatic mammals of the Brazilian Amazon disclosed a new group and a species-specific marker. Parasitology 2004;128:283-94.

27. Thorpe JP, Solé-Cava AM. The use of allozyme electrophoresis in invertebrate systematics. Zool Scr 1994;23:3-18

28. Brisse $\mathbf{S}$, Barnabé $\mathbf{C}$, Tibayrenc $\mathbf{M}$. Identification of six Trypanosoma cruzi phylogenetic lineages by random amplified polymorphic DNA and multilocus enzyme electrophoresis. Int J Parasitol 2000;30:35-44.

29. Brisse S, Verhoef J, Tibayrenc M. Characterization of large and small subunit rRNA and mini-exon genes further supports the distinction of six Trypanosoma cruzi lineages. Int J Parasitol 2001;31:1218-26. 